\title{
On 5G Radio Access Network Slicing: Radio Interface Protocol Features and Configuration
}

\author{
R. Ferrús, O. Sallent, J. Pérez-Romero, R. Agustí \\ Universitat Politècnica de Catalunya (UPC) \\ [ferrus, sallent, jorperez, ramon]@tsc.upc.edu
}

\begin{abstract}
Network slicing is a fundamental capability for future Fifth Generation (5G) networks to facilitate a cost-effective deployment and operation of multiple logical networks over a common physical network infrastructure in a way that each logical network (i.e. network slice) can be customized and dimensioned to best serve the needs of specific applications (e.g. mobile broadband, smart city, connected car, public safety, fixed wireless access) and users (e.g. general public, enterprise customers, virtual operators, content providers). The practical realization of such capability still raises numerous technical challenges, both in the Core and Radio Access Network (RAN) parts of the 5G system. Through a comprehensive analysis of the impact that the realization of RAN slicing has on the different layers of the radio interface protocol architecture, this paper proposes a framework for the support and specification of RAN slices based on the definition of a set of configuration descriptors that characterize the features, policies and resources to be put in place across the radio protocol layers of a next generation RAN node.
\end{abstract}

Keywords-Network slicing; RAN slicing; Multi-tenancy; RAN sharing; 5G networks, Radio Interface Protocol Architectures

\section{INTRODUCTION}

Unlike current 4G systems mainly designed to provide a 'one size fits all' mobile broadband solution, 5G systems are intended to simultaneously support a wider range of application scenarios and business models (e.g. automotive, utilities, smart cities, high-tech manufacturing) [1]. This expected versatility comes with a high variety of requirements on network functionalities (e.g. security, mobility, policy control features) and expected performance (e.g. peak rates above $10 \mathrm{Gbps}$, latencies below $1 \mathrm{~ms}$ with $10^{-5}$ reliability, $500 \mathrm{~km} / \mathrm{h}$ mobility target) that cannot always be met through a common network setting (e.g. optimizing the network for low latency with high reliability could come at the expenses of reduced spectral efficiency). In this context, support for network slicing in $5 \mathrm{G}$ systems has become a foundational requirement to allow $5 \mathrm{G}$ system operators to compose and manage dedicated logical networks with specific functionality, without 
losing the economies of scale of a common infrastructure [2]. Each one of these logical networks, referred to as network slice, can be tailored to fulfil at least a couple of purposes:

- To provide a particular system behavior (i.e. slice type) through the use of specific control plane (CP) and/or user plane (UP) functions to best support specific service/applications domains (e.g. optimized protocols for enhanced MBB [eMBB], massive Machine Type Communications [mMTC], UltraReliable and Low Latency Communications [URLLC]). For instance, a User Equipment (UE) for smart metering applications can be served through a network slice with radio access tailored to very small, infrequent messages and with no need to implement unnecessary functions (e.g. no mobility support).

- To provide a particular tenant (i.e. an organization or business entity entitled to use the network slice) with a given level of guaranteed network resources and isolation with regard to the operation of other concurrent slices. For instance, UEs/subscribers of a Public Safety (PS) agency can be served through a network slice that guarantees a minimum capacity during network congestion periods.

3GPP has recently completed the normative specifications regarding service and operational requirements to support network slicing [3] and work has started on both system architecture aspects [4] and related management and orchestration capabilities [5]. Simultaneously, the network slicing concept is being addressed in the 5G architectures currently under development in different research projects such as 5GNORMA [6], METIS-II [7] or SESAME [8]. Indeed, a complete solution for network slicing combines multiple facets, ranging from virtualization techniques for the abstraction and sharing of radio resources (e.g. network virtualization substrate concept in [9]) up to network slice lifecycle management solutions enabling the delivery of Network Slice as a Service (e.g. 5G network slice broker concept in [10]).

In this context, this paper elaborates on the realization of network slicing within the Radio Access Network (RAN), which still poses multiple open questions. To this end, the paper firstly tackles the overall network slicing architectural framework for $5 \mathrm{G}$ systems and identifies the fundamental design challenges for the realization of the RAN slicing. Then, a solution approach is proposed through the definition of a set of descriptors to parametrize the features, policies and resources within the radio protocol layers for the configuration of a RAN slice. The systematic and comprehensive analysis conducted is, to the authors' best knowledge, the first attempt to tangibly structure and specify how a RAN slice can be realized and how various RAN slices can be separately customized. Finally, the applicability of the proposed RAN slicing solution framework is illustrated. 


\section{Architectural Framework And RAN SLicing Design Challenges}

The realization of network slices considers, in the most general case, support for specific features and resources both in the $5 \mathrm{G}$ Core Network $(5 \mathrm{GC})$ part, which we will refer to as $\mathrm{CN}$ slice, and in the New Generation RAN (NG-RAN) part, referred to as RAN slice. A network slice is to be uniquely identified by a Slice_ID within a 5G network, the latter identified by a Public Land Mobile Network (PLMN) identity. The Slice_ID could take standard values to facilitate slicing configurations across networks in roaming scenarios or just remain PLMN-specific [4].

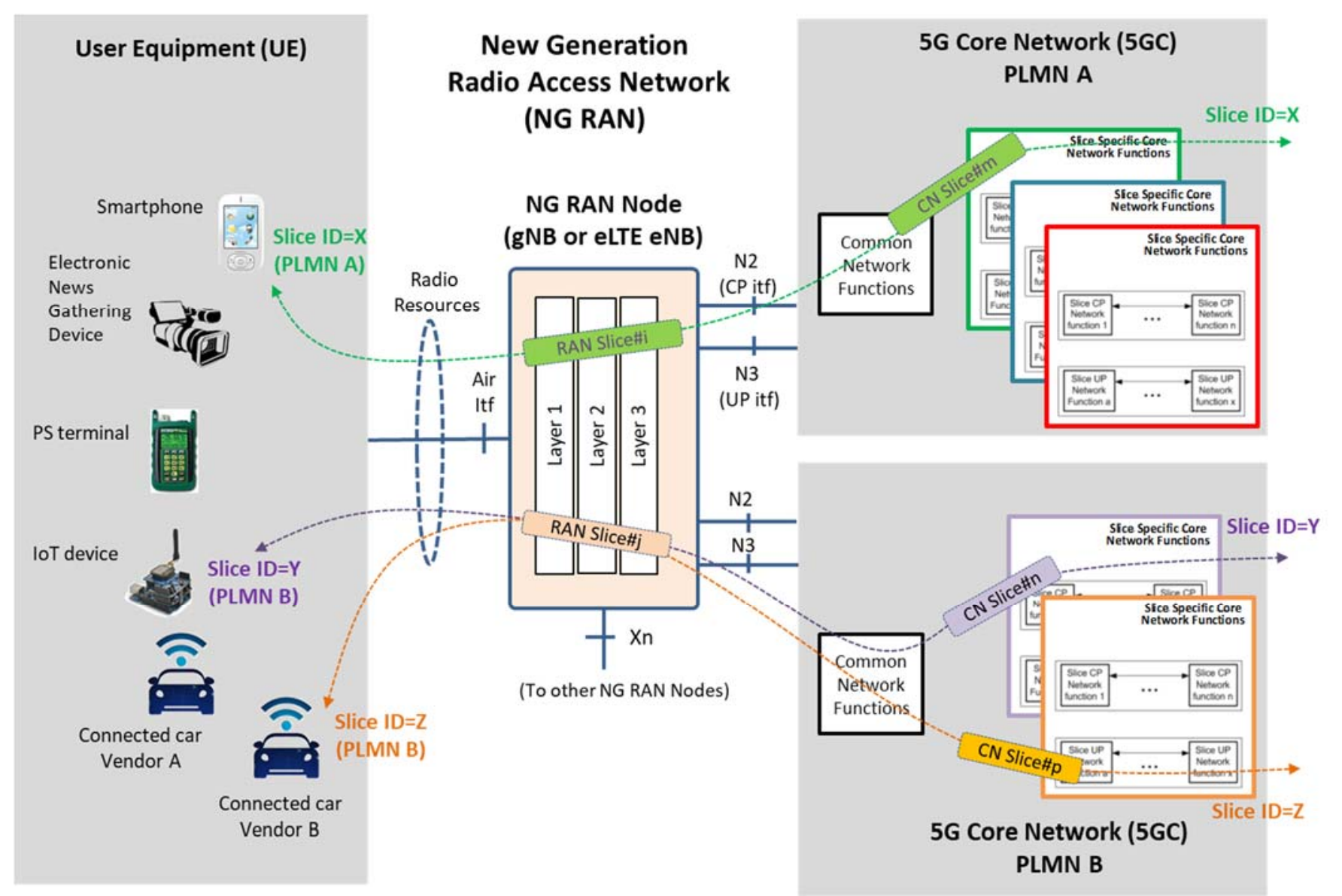

Fig. 1.- Illustration of the network slicing architecture

On the 5GC side, $\mathrm{CN}$ slices are to be realized through the deployment of a combination of a highly modularized set of 5GC functionality for CP and UP functions, including network functions (NFs) for e.g. network registration and mobility management (i.e. Access and Mobility Management Function [AMF]), 5G connectivity service handling (i.e. Session Management Function [SMF]), user plane forwarding and QoS handling (i.e. User Plane Function [UPF]), etc. As illustrated in Fig.1, some NF instances could be common to several $\mathrm{CN}$ slices, that is, supporting and logically belonging to more than one slice, while other $\mathrm{NF}$ instances just serving specific $\mathrm{CN}$ slices (e.g. the AMF instance, which conveys all the UE-5GC 
signaling for registration area management, UE reachability in idle state, etc., has to be the same instance for all the network slices serving a given UE [4]). All these 5GC NFs are likely to be implemented as virtualized network functions (VNFs) running on multi-tenant cloud infrastructures [11] and flexibly orchestrated as required.

On the other hand, the NG-RAN fundamentally consists of gNBs and/or eLTE eNBs, which are single NFs that provide the UP/CP protocol terminations towards the UEs and embed all the radio access functionality. Specifically, a gNB is a NG-RAN node operating the New Radio (NR) interface while a eLTE eNB is conceived as the evolution of the legacy eNB to support connectivity to both legacy CN (i.e. Evolved Packet Core [EPC]) and 5GC and so facilitate co-existence and migration options [12]. As illustrated in Fig. 1, a quite diverse range of UEs could be connected to the same NG-RAN node, though potentially served via different network slices. In this context, NFV technologies can also play a role in the realization of RAN slicing as long as a part of the RAN node functionality can be deployable as a VNF (e.g. a gNB node functionally split between a centralized unit, deployed with NFV, and a distributed unit with the specific RF components). However, regardless of the deployment options, the realization of the RAN slices requires addressing how the pool of radio resources (i.e. RF bandwidth) allocated to one NG RAN node can be configured and operated to simultaneously deliver multiple and diverse RAN behaviors, turning the RAN slicing support into a much more challenging issue. In this respect, let's consider that the RF bandwidth operated by a NG-RAN node can be flexibly arranged into a number of RF channels with diverse transmission bandwidths. A simple approach to support multiple RAN slices with slice-specific/optimized waveforms would be to implement each slice type on different cells using separate RF channels and radio protocol layer instances (i.e. Layer 1, 2 and 3 functions), similarly as done today with the multiple Radio Access Technologies (RATs) that co-exist in the current RAN infrastructures (e.g. UMTS cells, LTE cells, NB-IoT cells). However, scalability in terms of the number of supported slices types and reduced resource efficiency (i.e. no traffic multiplexing gains among slices) would be clearly major drawbacks. Therefore, the fundamental design challenge strives in the realization of multiple RAN slices that can be concurrently multiplexed in a single cell while at the same time achieving an efficient use of the radio resources. This entails tackling the following open questions:

- How to imbricate a number of slice-specific radio interface protocols over the same cell.

- How to manage the radio resource allocation to UEs within a cell so that pre-established levels on capacity and isolation can be offered per RAN slice.

- How to enable support for optimized Radio Resource Management (RRM) configurations and 
policies on a per-slice basis (e.g. admission policies, mobility control).

\section{REALIZATION OF RAN SLICES}

Taking as starting point the initial work conducted within 3GPP on NG-RAN and NR [12][13] as well as the radio interface protocol architecture already consolidated in current 3G/4G RANs, the analysis presented here shows that a set of new blocks of information, configuration descriptors and protocol features has to be introduced across the protocol layers of a NG-RAN node in order to address the mentioned open questions. In this respect, the proposed overall framework for RAN slicing support within a NG-RAN node is illustrated in Fig. 2 and explained in the following.

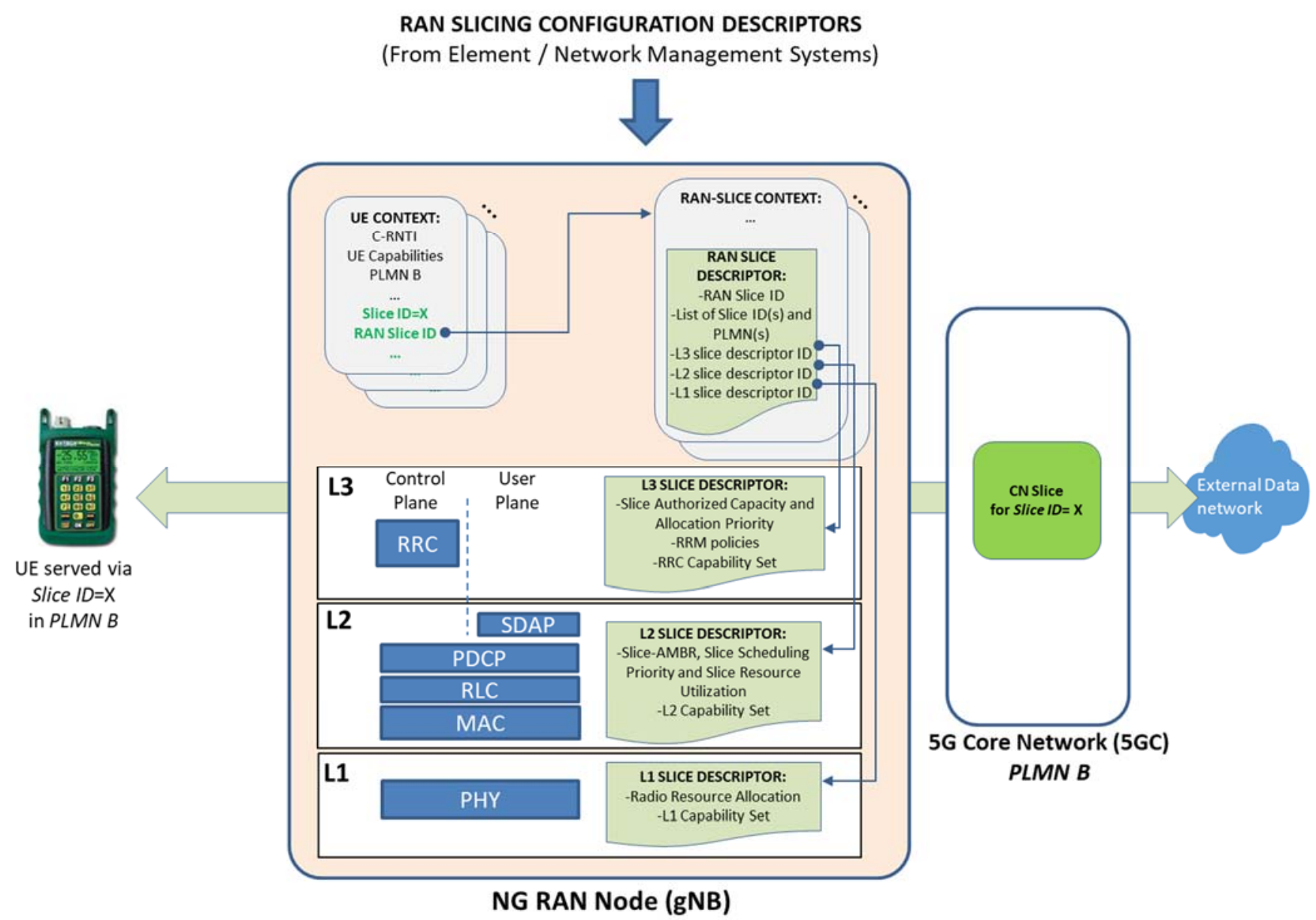

Fig. 2.- Framework for the realization of RAN slices in a NG-RAN node

From an operational perspective, a UE Context is instantiated within the RAN at the time that the UE becomes active and establishes a logical control connection (i.e. Radio Resource Control [RRC] connection). The UE Context, populated from information exchanged between the RAN and both the UE and $\mathrm{CN}$, is a block of information that contains all the necessary data required to maintain the RAN services 
towards the UE (e.g., temporary identifiers such as the Cell Radio Network Temporary Identity [C-RNTI], security context, UE capabilities, QoS related information, etc.). On this basis, we propose to add a RAN slice identifier (RAN_Slice_ID) to the UE Context and use it as a pointer to a new block of information within the NG-RAN node, denoted as RAN Slice Context, containing all the data necessary to support the operation of a particular RAN slice along with the Slice_ID(s) that are served through the RAN slice. The selection of the RAN_Slice_ID(s) is to be conducted by the NG-RAN node based on the Slice_ID(s) signaled by the UEs during the initial attach procedure or indicated from $\mathrm{CN}$ in subsequent signaling (the specific signaling procedures are still work in process within the 3GPP [4]).

Accordingly, from a functional perspective, it is proposed to specify the operation of each RAN slice through a set of configuration descriptors that parametrizes the features, policies and resources put in place across the radio protocol layers of the RAN node. Particularly, a RAN Slice Descriptor is introduced as the baseline descriptor for the instantiation of a RAN Slice Context within a RAN node. As illustrated in Fig. 2, the RAN Slice Descriptor includes at least the RAN_Slice_ID, the list of associated Slice_ID(s) and PLMN_ID(s), and a set of pointers to the configuration descriptors of the underlying radio protocol layers 3 , 2 and $1(\mathrm{~L} 3, \mathrm{~L} 2, \mathrm{~L} 1)$ for the realization of the RAN slice:

- L3 slice descriptor. L3 comprises the RRC protocol and RRM functions such as Radio Bearer Control (RBC), Radio Admission Control (RAC) and Connection Mobility Control (CMC) for the activation and maintenance of Radio Bearers (RB), which are the data transfer services delivered by the radio protocol stack. For each UE, one or more user plane RBs, denoted as Data RBs (DRBs), can be established per Protocol Data Unit (PDU) session, which defines the connectivity service provided by $5 \mathrm{GC}$ [12]. It's worth noting that traffic flows from different slices are not served by the same DRBs since traffic for different slices is handled through different PDU sessions [4]. Accordingly, a L3 slice descriptor is necessary to specify the capacity allocation for the RAN slice (e.g. number and characteristics of the DRBs that can be simultaneously established), the RRM policies that govern the operation of the slice (e.g. DRB configuration policies) and the capability set of the RRC protocol in use (e.g. application type specific RRC messages).

- L2 slice descriptor. L2 comprises a Medium Access Control (MAC) sub-layer for multiplexing and scheduling the packet transmissions of the DRBs over a set of transport channels exposed by L1. Moreover, L2 embeds a number of processing functions configurable on a per-DRB basis for e.g. segmentation, Automatic Repeat reQuest (ARQ) retransmissions, compression and ciphering (i.e. Radio Link Control [RLC] and Packet Data Convergence Protocol [PDCP]). In the NR specifications, 
an additional L2 sub-layer named Service Data Adaptation Protocol (SDAP) is included to map the DRBs and the traffic flows managed by the $5 \mathrm{GC}$, referred to as QoS Flows [12]. Therefore, considering that the current MAC operation is based on individual UE and DRB -specific QoS profiles, a L2 slice descriptor is necessary to define the packet scheduling behaviors to be enforced on the traffic aggregate of DRBs of the same slice and to specify the capability set of the applicable L2 sub-layers processing functions.

- L1 slice descriptor. L1 provides L2 with transfer services in the form of transport channels, which define how the data is transferred (e.g. Transmission Time Interval [TTI], channel coding). L1 also establishes the corresponding radio resource structure of the cell radio resources (e.g. waveform characteristics and time-/frequency- domain resource structure). Considering that a RAN slice may require specific L1 transfer service capabilities (e.g. low latency shared transport channel) and/or specific radio resource allocation of the cell radio resources, a L1 slice descriptor is needed to specify both aspects.

More details on the above introduced descriptors and their impact on the configuration and extended features necessary for RAN slicing within L3, L2 and L1 are discussed below.

\section{A. L3 configuration}

When multiple RAN slices are realized over shared radio resources, the RRM functions for RBC, RAC and CMC have to assure that each RAN slice gets the expected amount of resources and, in case, handle any resource conflicts that might appear across slices. These RRM functions are typically implemented as vendor-specific algorithms only abided by the use of the data models and control signaling capabilities established in the standards (e.g. UE and RB QoS model parameters, RRC protocol messages and procedures). Therefore, we propose to specify the following set of parameters per RAN slice to dictate the operation of the RRM functions for capacity allocation and traffic isolation among the slices:

- Slice Authorized Capacity. This can be a combination of resource-oriented and rate-oriented parameters that limit the number and characteristics of the RBs established for the entire slice. Resource-oriented parameters can include absolute or relative occupation levels of the consumed radio resources (i.e. radio resource limitations) as well as hard limits on the number of simultaneously established UE Contexts/RBs (i.e. license limitations). Rate-oriented parameters can include rate limits on the aggregate bit rate of the entire set of admitted Guaranteed Bit Rate (GBR) RBs within the slice. All these slice capacity parameters are to be used by the RAC for the admission/rejection of $\mathrm{RBs}$ as well as by the RBC/CMC functions in order to decide on modifications, handovers or even 
forced releases of active RBs if network dynamics turns into increased radio resource consumption in excess of the established limits.

- Slice Allocation Priority. This parameter allows for conflict resolution amongst UE/RB resource requirements across slices that cannot be solved based only on the Slice Authorized Capacity parameters nor using the policies associated with the individual UE/RBs (e.g. a situation in which there are two GBR DRB admission requests with the same QoS profile in distinct RAN slices that cannot be granted simultaneously due to temporary congestion). In legacy 3G/4G RANs, priority and pre-emption policies at UE/RB level are solved through the Allocation and Retention Priority (ARP) parameter included in the QoS profile. The ARP encodes information about priority level (scalar with 15 levels), pre-emption capability (flag with “yes" or "no") and pre-emption vulnerability (flag “yes" or "no"). A similar semantic can be adopted for the Slice Allocation Priority parameter.

Moreover, since pursuing different optimization targets for the efficient use of the radio resources requires RRM functions with different parameterization, the RRM policies should be specifiable on a perslice basis. This can be accomplished through a prescriptive specification, establishing the RRM algorithms and associated configuration parameters (e.g. thresholds, timers), and/or a declarative specification, establishing the expected RRM behavior (e.g. Key Performance Indicators [KPIs] and optimization goals).

Regarding the RRC protocol, the customization of RRC messages and procedures on a per-slice basis (e.g. specific RRC signaling for Narrow Band - Internet of Things [NB-IoT] applications) can be realized without any impact on the protocol stack if customized RRC signaling is only used through UE-dedicated Signaling RBs (SRBs) and/or through common logical channels not shared with other RAN slices. However, if multiple RAN slices are configured to share the same set of common logical channels (e.g. Broadcast Control Channel [BCCH], Paging Control Channel [PCCH] and Common Control Channels $[\mathrm{CCCH}])$, the following extended features have to be incorporated within the RRC protocol:

- Protocol fields within the RRC messages to allow UEs to discriminate among signaling from different slices.

- System Information Block (SIB) messages to advertise through a common BCCH the Slice_ID(s) that can be reached from the cell. This allows the UE to take into account this information for network discovery and selection processes.

- SIB messages to support cell (re-)selection parameters and neighboring cell information broadcasting per Slice_ID, so that the behavior of terminals in idle mode can be set differently per-slice. 
- SIB messages to support Access Barring and Load Control (AB/LC) per Slice_ID so that unscheduled transmissions over the uplink $\mathrm{CCCH}$ can be controlled separately per slice.

- Paging configuration features allowing paging cycles to be organized considering the specifics needs of each Slice_ID.

\section{B. L2 configuration}

In current $3 \mathrm{G} / 4 \mathrm{G}$ systems, radio resource scheduling by the MAC takes account of the traffic volume and radio conditions per UE together with the UE/RB QoS parameters that define the expected forwarding behavior (e.g. QoS Class Identifier [QCI], GBR, Maximum Bit Rate [MBR], Aggregated MBR per UE [UEAMBR]). While this approach suffices for QoS differentiation on the packet forwarding treatment per$\mathrm{UE} / \mathrm{RB}$, it lacks of any formalization to parameterize the expected QoS behavior at packet forwarding level for the traffic aggregate within a given RAN slice.

Therefore, the proposed L2 slice descriptor includes the following parameters to dictate, along with the per-UE/RB QoS profiles, the operation of the MAC scheduler and yield isolation at packet forwarding level on a per-slice basis:

- Slice-AMBR, to limit the aggregate bit rate of all the Non-GBR RBs associated with the slice.

- Slice Scheduling Priority, to handle short-term traffic congestion conflicts between RBs with the same QoS profile (e.g. same QCI) but belonging to slices that should be given different precedence treatment.

- Slice Resource Utilization, used to establish constraints on the amount of physical layer resources scheduled by the MAC that are consumed by the slice. This constraint can be formulated as a percentage of the overall L1 resources that are managed by the MAC scheduler.

In addition, the L2 slice descriptor includes a L2 capability set to establish the possible configuration options for the RBs associated with the slice, including Hybrid ARQ configurations (e.g. synchronous / asynchronous operation and number of processes in parallel), RLC operation modes (e.g. acknowledged/unacknowledged/transparent modes and status reporting) and PDCP options (e.g. ciphering support).

\section{L1 configuration}

The new physical layer for 5G NR [13] is being defined with the target to provide high flexibility for the use of different waveforms (e.g. orthogonal frequency-division multiplexing [OFDM]-based waveforms 
with different numerologies) and adaptable time-frequency frame structures (e.g. selectable slot durations, dynamic assignment of DL/UL transmission direction). Considering that the L1 optimal settings can differ per slice type, the proposed L1 descriptor intends to establish a partitioning of the L1 radio resource structure so that different L1 optimization settings can be simultaneously applied.

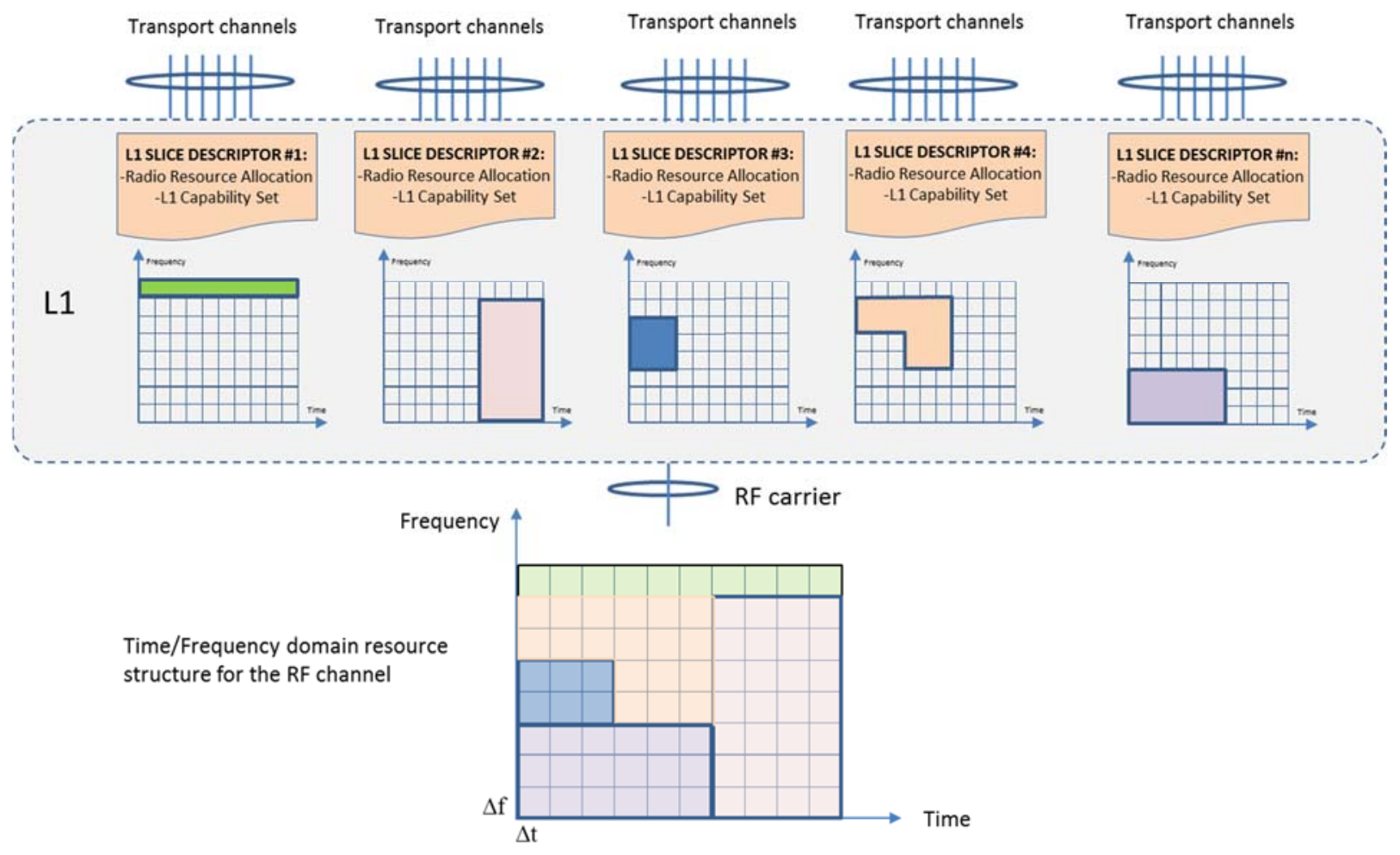

Fig. 3.- Illustration of slicing support in L1

The proposed approach is illustrated in Fig. 3. Each tile represents the smallest allocation unit of time duration $\Delta \mathrm{t}$ and frequency size $\Delta \mathrm{f}$ (e.g. $\Delta \mathrm{t}$ and $\Delta \mathrm{f}$ could be an integer number of, respectively, OFDM symbols and subcarriers with the minimum supported subcarrier separation). On this basis, the overall RF carrier resources are split off in a number of $L 1$ slices that could be separately optimized to offer specific transfer service capabilities (e.g. different TTI sizes, synchronous/asynchronous access). The mixing of L1 slices in the same resource grid with different waveforms characteristics could be achieved through the use of the different OFDM numerologies and transmitter windowing techniques that mitigate the potential interslice interference [14]. Moreover, this segmentation of resources per slice avoids the transmission of common reference and control signals over the entire RF bandwidth but instead use self-contained transmissions per slice that can be arranged as needed (e.g. one slice could be configured to use a strict time-division separation of the physical layer control and data, like in current LTE, while in another slice control and data could be multiplexed over the same radio resources [15]). Note that this approach requires 
some minimum amount of resources to be allocated within the resource grid structure to facilitate the cell search process (e.g. common reference signals for UEs to acquire time and frequency synchronization) and provide the information for UEs to locate the control channels within the L1 slices. This approach also allows the segmentation of resources to be dynamic (e.g. the location and number of resource units allocated to each L1 slice adapted over time to match traffic demand variations).

\section{RAN SLICING CONFIGURATION EXAMPLES}

To gain insight into the proposed framework, let us consider an illustrative scenario for the applicability of RAN slicing involving multiple service providers, service types and groups of subscribers/applications. Let us assume a commercial MNO (MNO\#A - PLMN\#A) that has deployed a NG-RAN and primarily exploits it to deliver eMBB and IoT services to retail customers. To this end, MNO\#A sets up three RAN slices: one for eMBB and two for mMTC. The eMBB slice $\left(R A N \_S l i c e \_I D=1\right)$ is used for general public mobile broadband services, one of the mMTC slices $\left(R A N \_S l i c e \_I D=3\right)$ for small enterprise customers with IoT needs (e.g. transportation companies) and the other mMTC slice $\left(R A N \_S l i c e \_I D=4\right)$ for a few customers with large scale deployments of IoT applications (e.g. utilities). In addition, MNO\#A provides wholesale access to two 3rd party service providers, leasing capacity from its NG-RAN infrastructure. One 3rd party provider is a PS communications operator (MNO\#B) that uses the contracted RAN slice (RAN_Slice_ID=2) for Mission Critical Push-To-Talk (MCPTT) and MCVideo services in a way that supplements the capacity of its own dedicated PS broadband network (PLMN\#B). The second provider is a IoT communications provider (MNO\#C - PLMN\#C) specialized within the automotive sector, who exploits the leased RAN slice $\left(R A N \_S l i c e \_I D=5\right)$ customized to serve the needs of connected car applications.

Fig.4 provides a detailed illustrative view of the radio interface protocol architecture and the corresponding descriptors for the realization of this scenario. The overall configuration consists of five RAN Slice Descriptors, including a combination of five L3, four L2 and three L1 Slice Descriptors. At L1, L1_Slice_ID=1 is configured for eMBB services (based on e.g. a OFDM waveform as currently used for LTE), L1_Slice_ID=2 for mMTC services (based on e.g. the legacy NB-IoT physical layer) and L1_Slice_ID $=3$ for the delivery of URLLC services (based on e.g. a new 5G waveform optimized for vehicle-to-infrastructure communications, denoted as Car IoT in this example). A common frame duration of $10 \mathrm{~ms}$ is considered for the three L1 slices while the slot duration within each slice depends on the used OFDM numerology. Note that with NR it is expected that a UE may be instructed to receive or transmit using a subset of the resource grid only [13]. Moving upwards in the protocol stack, the two RAN slices 
( $R A N \_S l i c e \_I D=1$ and 2 ) on top of the $L 1 \_S l i c e \_I D=1$ are configured to use the same MAC instance (i.e. MAC-eMBB) and the same set of common logical channels (i.e. $\mathrm{BCCH}, \mathrm{PCCH}$ and $\mathrm{CCCH}$ ). Traffic isolation between $R A N \_S l i c e \_I D=1$ and 2 is enforced at both L2 and L3 levels through separate L2 and L3 slice descriptors. Like the two RAN slices for eMBB, the two slices for IoT services $\left(R A N \_S l i c e \_I D=3\right.$ and $\left.R A N \_S l i c e \_I D=4\right)$ are also configured to use a shared MAC instance (i.e MAC-NB-IoT) and common logical channels. However, isolation is now only configured at L3 level (i.e. both slices uses the same L2 configuration descriptor). Finally, it can be noted that the Car IoT L1 slice is configured to be only exploited by $R A N \_$Slice_ID $=5$, so that there is no need for isolation at L2 and L3.

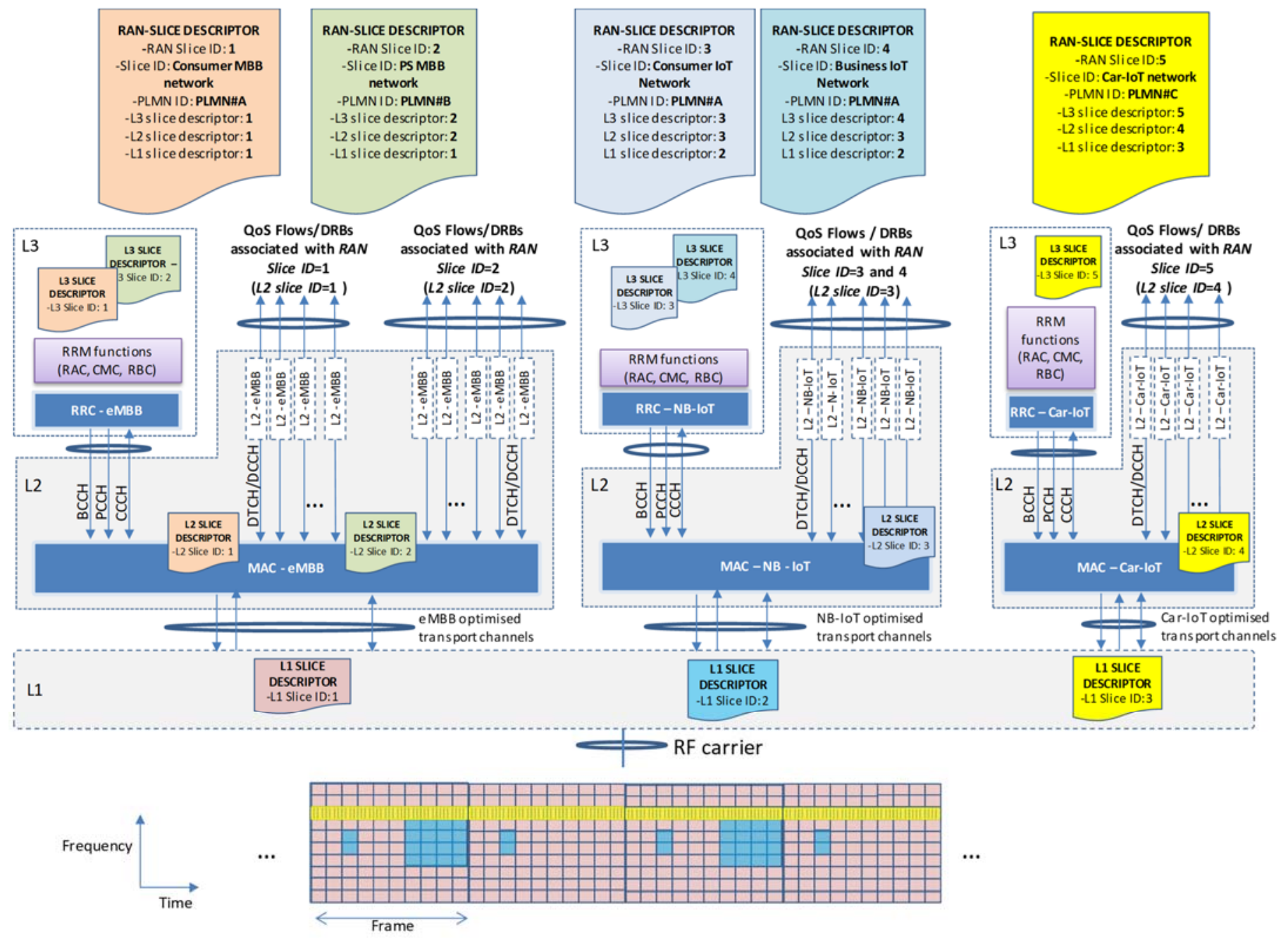

Fig. 4.- Radio protocol stack view of the RAN slicing configuration

Illustrative evaluation results are provided next to show how some L1, L2 and L3 configuration descriptors parameters impact on the achieved performance and level of isolation across the RAN slices. The evaluation scenario considers an outdoor urban micro cell operating in the 3.4-3.8 GHz frequency band 
with a carrier bandwidth of $100 \mathrm{MHz}$ (equivalent to 500 Physical Resource Blocks [PRBs] for an OFDM numerology with $\Delta \mathrm{f}=15 \mathrm{kHz}$ ). Table 1 describes the simulation settings including traffic demand and configuration parameters of each RAN slice. Of note is that, regarding the two eMBB slices, the Slice Authorized Capacity parameter within L3 slice descriptors is used to limit both the maximum aggregated bit rate of the active GBR bearers allowed per slice and the average number of PRBs consumed by this traffic. Moreover, the parameter Slice Resource Utilization within L2 slice descriptors is used to limit the maximum share per RAN slice of the PRBs used to serve Non-GBR bearers (i.e. L1_Slice_ID=1 resources not consumed by the GBR bearers). On the other hand, regarding the two mMTC slices, the parameter Slice Authorized Capacity is set to only limiting the number of connected devices. On this basis, key performance indicators achieved for the eMBB and mMTC slices are given in Table 2, where $t_{0}$ indicates a time instant under the traffic demand conditions in Table 1 and subsequent time instants consider some traffic demand variations. As seen in Table 2, GBR traffic overload in $R A N \_S l i c e \_I D=1$ from $\mathrm{t}_{1}$ onwards is mitigated via admission control as soon as GBR traffic starts compromising the Slice Authorized Capacity configuration so that GBR traffic served through $R A N \_$Slice_ID $=2$ remains unaffected. Note however that this GBR traffic increase leads to a reduction of the capacity left for Non-GBR traffic and, consequently, Non-GBR DRB rates are lowered in both $R A N \_S l i c e \_I D=1$ and 2 . On the other hand, the increase of Non-GBR connections in $R A N \_S l i c e \_I D=1$ from $t_{5}$ onwards, while it reduces Non-GBR DRB bit rates in $R A N \_S l i c e \_I D=1$, it does not hinder the Non-GBR DRB performance in $R A N \_S l i c e \_I D=2$ thanks to the protection achieved through the L2 descriptor. Note also that IoT applications performance remains unaffected from $t_{1}$ to $t_{6}$ because of the isolation enforced between $L 1$ slices. Finally, it can be seen that the smart meter application upgrade in $R A N \_$Slice_ID $=4$ leads to a delay increase for all IoT applications, including those running over $R A N \_S l i c e \_I D=3$ since the only isolation mechanism between $R A N \_$Slice_ID $=3$ and 4 is based on the limit of the number of connected devices, which remains unchanged. This situation could be avoided by either introducing L2 descriptors to limit the traffic served per slice or by dynamically up scaling L1_Slice_ID=2 from 1 to 2 PRB and down scaling L1_Slice_ID=1 from 450 to 449 $\mathrm{PRB}$ with a negligible impact on the eMBB slices.

\begin{tabular}{|l|l|l|}
\hline Parameter & Value \\
\hline $\begin{array}{l}\text { Deployment } \\
\text { settings }\end{array}$ & Path loss model & $\begin{array}{l}\text { Urban micro-cell model with hexagonal layout (source: Report ITU-R } \\
\text { M.2135 "Guidelines for evaluation of radio interface technologies for IMT- } \\
\text { Advanced") }\end{array}$ \\
\cline { 2 - 3 } & Shadowing standard deviation & $3 \mathrm{~dB}$ in LOS and 4 dB in NLOS. \\
\cline { 2 - 3 } & Base station antenna gain & $5 \mathrm{~dB}$ \\
\cline { 2 - 3 } & Frequency & $\begin{array}{l}3.6 \mathrm{GHz} \text { (source: RSPG16-032 FINAL "Europe Radio Spectrum Policy } \\
\text { Group Opinion on spectrum for 5G") }\end{array}$ \\
\cline { 2 - 3 } & Transmitted power per PRB & $14 \mathrm{dBm}$ \\
\hline
\end{tabular}




\begin{tabular}{|c|c|c|}
\hline & Carrier bandwidth & $100 \mathrm{MHz}$, equivalent to 500 PRBs for OFDM numerology with $\Delta \mathrm{f}=15 \mathrm{kHz}$ \\
\hline & UE noise figure & $9 \mathrm{~dB}$ (considered the same for all type of devices) \\
\hline & $\begin{array}{l}\text { Spectral efficiency model to } \\
\text { map SINR with bit rate }\end{array}$ & $\begin{array}{l}\text { L1_Slice_ID }=1 \text { : Model A.1 in } 3 \mathrm{GPP} \text { TR } 36.942 \text { and assuming a maximum } \\
\text { spectral efficiency of } 8.8 \mathrm{~b} / \mathrm{s} / \mathrm{Hz} \\
\text { L1_Slice_ID }=2 \text { and } 3: 1 \mathrm{~b} / \mathrm{s} / \mathrm{Hz}\end{array}$ \\
\hline \multirow[t]{4}{*}{ RAN_Slice_ID $=1$} & Traffic Demand & $\begin{array}{l}\text { Mix of GBR and Non-GBR traffic, modelled with Poisson session arrival } \\
\text { time distribution and exponential session duration. GBR DRBs used to offer } \\
10 \mathrm{Mb} / \mathrm{s} \text { high-quality video streaming services. } \\
\text { Average of } 100 \mathrm{Mb} / \mathrm{s} \text { for GBR traffic. } \\
\text { Average of } 10 \text { simultaneous active DRBs for Non-GBR traffic. }\end{array}$ \\
\hline & $\begin{array}{l}\text { L3 Descriptor - Slice } \\
\text { Authorized Capacity }\end{array}$ & $\begin{array}{l}\text { Maximum aggregated GBR traffic }=240 \mathrm{Mb} / \mathrm{s} \text { or up to } 56 \% \text { of PRBs } \\
\text { consumed on average in L1_Slice_ID }=1\end{array}$ \\
\hline & $\begin{array}{l}\text { L2 Descriptor - Slice Resource } \\
\text { Utilization }\end{array}$ & Up to $70 \%$ of the PRBs left available for Non-GBR traffic in $L 1$ Slice_ID $=1$ \\
\hline & $\begin{array}{l}\text { L1 Descriptor - Radio resource } \\
\text { allocation }\end{array}$ & 450 PRB (shared with $R A N \_S l i c e \_I D=2$ ) \\
\hline \multirow[t]{4}{*}{ RAN_Slice_ID $=2$} & Traffic Demand & $\begin{array}{l}\text { Mix of GBR and Non-GBR traffic, modelled with Poisson session arrival } \\
\text { time distribution and exponential session duration. GBR DRBs used for } \\
\text { MCVideo with } 2 \mathrm{Mb} / \mathrm{s} \text { standard video quality. } \\
\text { Average of } 30 \mathrm{Mb} / \mathrm{s} \text { for GBR traffic. } \\
\text { Average of } 5 \text { simultaneous active DRBs for Non-GBR traffic. }\end{array}$ \\
\hline & $\begin{array}{l}\text { L3 Descriptor - Slice } \\
\text { Authorized Capacity }\end{array}$ & $\begin{array}{l}\text { Maximum Aggregated GBR }=60 \mathrm{Mb} / \mathrm{s} \text { or up to } 14 \% \text { of PRBs consumed on } \\
\text { average in L1_Slice_ID }=1\end{array}$ \\
\hline & $\begin{array}{l}\text { L2 Descriptor - Slice Resource } \\
\text { Utilization }\end{array}$ & Up to $50 \%$ of the PRBs left available for Non-GBR traffic in $L 1$ Slice_ID $=1$ \\
\hline & $\begin{array}{l}\text { L1 Descriptor - Radio resource } \\
\text { allocation }\end{array}$ & 450 PRB (shared with $R A N \_S l i c e \_I D=1$ ) \\
\hline \multirow[t]{3}{*}{$R A N \_S l i c e \_I D=3$} & Traffic Demand & $\begin{array}{l}\text { Mix of IoT applications (e.g. assets tracking, environmental monitoring, etc.) } \\
\text { whose traffic aggregate is characterised by messages of } 128 \text { Bytes every } 1 \\
\text { minute on average. Total number of connected devices is } 1000 \text {. }\end{array}$ \\
\hline & $\begin{array}{l}\text { L3 Descriptor - Slice } \\
\text { Authorized Capacity }\end{array}$ & Maximum number of UE Contexts $=2000$ \\
\hline & $\begin{array}{l}\text { L1 Descriptor - Radio resource } \\
\text { allocation }\end{array}$ & 1 PRB (shared with $R A N \_S l i c e \_I D=4$ ) \\
\hline \multirow[t]{3}{*}{ RAN_Slice_ID $=4$} & Traffic Demand & $\begin{array}{l}\text { Smart meter application that generates } 1024 \text { Bytes messages every } 2 \\
\text { minutes. Total number of connected devices is } 400 \text {. }\end{array}$ \\
\hline & $\begin{array}{l}\text { L3 Descriptor - Slice } \\
\text { Authorized Capacity }\end{array}$ & Maximum number of UE Contexts $=500$ \\
\hline & $\begin{array}{l}\text { L1 Descriptor - Radio resource } \\
\text { allocation }\end{array}$ & 1 PRB (shared with $R A N \_S l i c e \_I D=3$ ) \\
\hline \multirow[t]{2}{*}{ RAN_Slice_ID $=5$} & Traffic Demand & Full buffer model \\
\hline & $\begin{array}{l}\text { L1 Descriptor - Radio resource } \\
\text { allocation }\end{array}$ & 49 PRB \\
\hline
\end{tabular}

Table 1. Simulation parameters

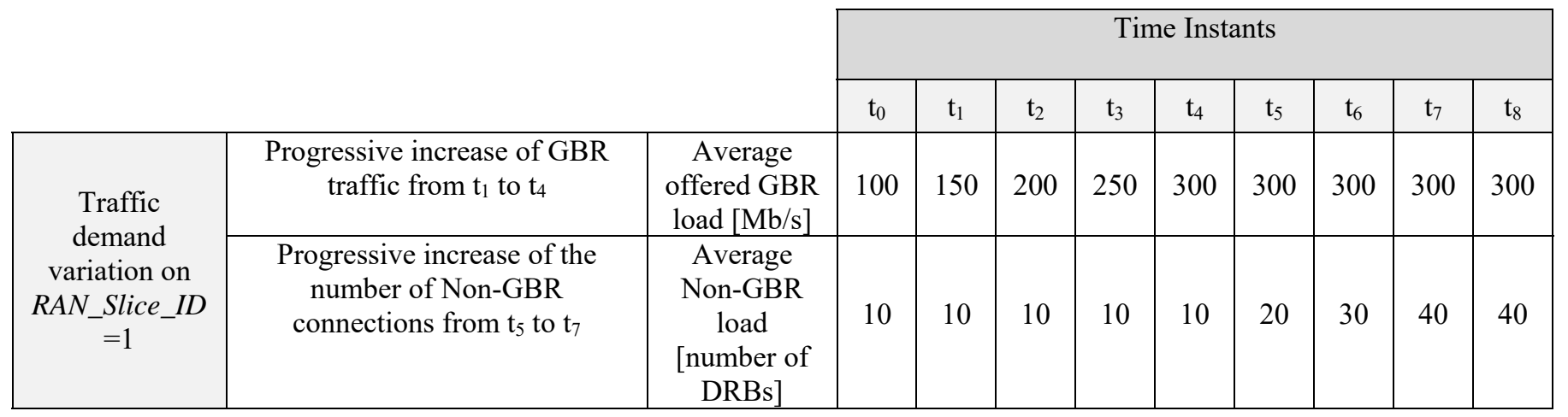




\begin{tabular}{|c|c|c|c|c|c|c|c|c|c|c|c|}
\hline $\begin{array}{c}\text { Traffic } \\
\text { demand } \\
\text { variation on } \\
R A N \_S l i c e \_I D \\
=4\end{array}$ & $\begin{array}{l}\text { Periodic reporting decrease due } \\
\text { to smart meter application } \\
\text { upgrades implemented in two } \\
\text { phases }\left(\mathrm{t}_{7} \text { and } \mathrm{t}_{8}\right) \text {. No change in } \\
\text { the number of connected } \\
\text { devices. }\end{array}$ & $\begin{array}{c}\text { Message } \\
\text { periodicity } \\
{[\mathrm{s}]}\end{array}$ & 120 & 120 & 120 & 120 & 120 & 120 & 120 & 60 & 30 \\
\hline \multirow{6}{*}{$\begin{array}{l}\text { Performance } \\
\text { indicators }\end{array}$} & \multicolumn{2}{|c|}{$\begin{array}{l}R A N \_ \text {Slice_ID }=1-\text { Admission Acceptance Ratio } \\
\text { for GBR DRBs }[\%]\end{array}$} & 99.99 & 99.23 & 93.81 & 84.06 & 74.30 & 65.42 & 65.45 & 65.32 & 65.40 \\
\hline & \multicolumn{2}{|c|}{$\begin{array}{l}R A N \_ \text {Slice_ID }=2 \text { - Admission Acceptance Ratio } \\
\text { for GBR DRBs [\%] }\end{array}$} & 99.97 & 99.99 & 99.93 & 99.97 & 99.99 & 99.94 & 99.92 & 99.96 & 99.91 \\
\hline & \multicolumn{2}{|c|}{$\begin{array}{l}R A N \_S l i c e \_I D=1 \text { - Average rate per Non-GBR } \\
\text { DRB }[\mathrm{Mb} / \mathrm{s}]\end{array}$} & 21.7 & 18.4 & 15.6 & 14.1 & 13.2 & 6.9 & 4.6 & 3.4 & 3.4 \\
\hline & \multicolumn{2}{|c|}{$\begin{array}{l}R A N \_ \text {Slice_ID }=2 \text { - Average rate per Non-GBR } \\
\mathrm{DRB}[\mathrm{Mb} / \mathrm{s}]\end{array}$} & 21.7 & 18.4 & 15.6 & 14.1 & 13.2 & 11.9 & 11.9 & $\mid 11.8$ & 11.9 \\
\hline & \multicolumn{2}{|c|}{$\begin{array}{l}\text { RAN_Slice_ID=3 - Average Message Queueing } \\
\text { Delay [ms] }\end{array}$} & 12.8 & 12.7 & 12.7 & 12.6 & 12.9 & 12.7 & 12.7 & 23.2 & 125.1 \\
\hline & \multicolumn{2}{|c|}{$\begin{array}{l}\text { RAN_Slice_ID=4 - Average Message Queueing } \\
\text { Delay [ms] }\end{array}$} & 52.4 & 52.3 & 52.4 & 52.4 & 52.2 & 52.3 & 52.4 & 62.5 & 165.5 \\
\hline
\end{tabular}

Table 2. Illustrative assessment of the degree of isolation between the RAN slices

\section{CONCLUDING REMARKS}

This article has described a framework that establishes the new blocks of information, configuration descriptors and extended protocol features to be introduced within a NG-RAN node for the realization of RAN slicing, thus enabling multiple RAN slices, with potentially different radio protocol behaviors as well as different levels of guaranteed network resources and isolation, to be concurrently multiplexed over the same cell. Remarkably, the proposed configuration descriptors provide a functional characterization of the RAN slice by parametrizing the features, policies and resources in place across L3, L2 and L1 protocol layers of the radio interface. An illustrative use case has been developed in detail to show the applicability of the proposed framework, visualizing the resulting radio interface protocol architecture along with the specific parameters involved in the RAN slicing configuration descriptors.

\section{ACKNOWLEDGEMENTS}

This work has been supported by the EU funded H2020 5G-PPP project 5G ESSENCE under the grant agreement 761592 and by the Spanish Research Council and FEDER funds under RAMSES grant (ref. TEC2013-41698-R).

\section{REFERENCES}

[1] NGMN Alliance, “5G White Paper", February 2015.

[2] 3GPP TR 22.864: "Feasibility Study on New Services and Markets Technology Enablers - Network Operation; Stage 1 (Release 15)", September 2016. 
[3] 3GPP TS 22.261 v15.0.0, “Service requirements for the 5G system; Stage 1 (Release 15)”, March 2017.

[4] 3GPP TS 23.501 V1.0.0, "System Architecture for the 5G System; Stage 2 (Release 15)”, June 2017.

[5] 3GPP TR 28.801 V1.2.0, "Study on management and orchestration of network slicing for next generation network (Release 15)”, May 2017.

[6] P. Rost et al., "Mobile network architecture evolution toward 5G," in IEEE Communications Magazine, vol. 54, no. 5, pp. 84-91, May 2016.

[7] METIS II White Paper "Preliminary Views and Initial Considerations on 5G RAN Architecture and Functional Design", March 2016.

[8] O. Sallent; J. Perez-Romero; R. Ferrús; R. Agusti, "On Radio Access Network Slicing from a Radio Resource Management Perspective" to appear in IEEE Wireless Communications, April 2017

[9] X. Costa-Perez, J. Swetina, T. Guo, R. Mahindra and S. Rangarajan, "Radio access network virtualization for future mobile carrier networks," in IEEE Communications Magazine, vol. 51, no. 7, pp. 27-35, July 2013

[10] K. Samdanis, X. Costa-Perez and V. Sciancalepore, "From network sharing to multi-tenancy: The 5G network slice broker," in IEEE Communications Magazine, vol. 54, no. 7, pp. 32-39, July 2016.

[11] V. Del Piccolo, A. Amamou, K. Haddadou and G. Pujolle, "A Survey of Network Isolation Solutions for Multi-Tenant Data Centers," in IEEE Communications Surveys \& Tutorials, vol. 18, no. 4, pp. 2787-2821, Fourthquarter 2016.

[12] 3GPP TS 38.300 V0.4.1, "NR; NR and NG-RAN Overall Description; Stage 2 (Release 15)", June 2017.

[13] 3GPP TS 38.211 V0.1.0, "NR; Physical channels and modulation (Release 15)", June 2017.

[14] A. A. Zaidi et al., "Waveform and Numerology to Support 5G Services and Requirements," in IEEE Communications Magazine, vol. 54, no. 11, pp. 90-98, November 2016.

[15] K. I. Pedersen, G. Berardinelli, F. Frederiksen, P. Mogensen and A. Szufarska, "A flexible 5G frame structure design for frequency-division duplex cases," in IEEE Communications Magazine, vol. 54, no. 3, pp. 53-59, March 2016. 